\title{
Analyse environnementale de la planification provinciale et territoriale aux fins des programmes de vaccination contre la COVID-19 au Canada
}

\author{
Shannon MacDonald ${ }^{1 *}$, Hannah Sell ${ }^{1,2}$, Sarah Wilson ${ }^{3}$, Samantha Meyer ${ }^{4}$, Arnaud Gagneur ${ }^{5}$, \\ Ali Assi ${ }^{1}$, Manish Sadarangani ${ }^{6}$, et les membres du groupe d'étude COVImm
}

Contexte : Les services de santé publique du Canada sont actuellement confrontés à la tâche difficile de planifier et de mettre en œuvre des programmes de vaccination contre la maladie à coronavirus 2019 (COVID-19).

Objectif : Recueillir et synthétiser les informations concernant la planification du programme de vaccination contre la COVID-19 dans chaque province et territoire du Canada, y compris les considérations logistiques, les groupes prioritaires et la surveillance de l'innocuité et de l'efficacité du vaccin.

Méthodes : Les responsables provinciaux et territoriaux de la santé publique ont été interrogés par téléconférence au cours de la phase initiale de planification des programmes de vaccination contre la COVID-19 (août à octobre 2020) afin de recueillir des informations sur les sujets suivants : facteurs uniques pour la vaccination contre la COVID-19, intention d'adopter les recommandations du Comité consultatif national de l'immunisation (CCNI), groupes prioritaires pour les premières phases de la vaccination et surveillance de l'innocuité et de l'efficacité du vaccin. Les données ont été regroupées en fonction des réponses communes et une analyse descriptive a été réalisée.

Résultats : Dix-huit entretiens ont eu lieu avec 25 participants de 11 des 13 provinces et territoires. Les facteurs uniques de la vaccination contre la COVID-19 comprenaient la hiérarchisation des groupes pour les premières phases de la vaccination $(n=7)$, la perception des vaccins par le public $(n=6)$ et des critères d'éligibilité divergents $(n=5)$. Presque toutes les provinces et territoires $(n=10)$ ont déclaré s'appuyer sur les recommandations du CCNI. Les résidents des établissements de soins de longue durée $(n=10)$ et les travailleurs de la santé $(n=10)$ ont été le plus souvent prioritaires pour les premières phases de la vaccination, suivis des personnes souffrant de maladies chroniques $(n=9)$ et des personnes âgées $(n=8)$. La plupart des provinces et territoires $(n=9)$ prévoient une surveillance systématique des effets indésirables pour évaluer l'innocuité des vaccins. L'évaluation de l'efficacité devait se faire au sein des services de santé publique $(n=3)$, par des chercheurs $(n=3)$ ou sur la base de directives nationales $(n=4)$.

Conclusion : La planification des programmes de vaccination contre la COVID-19 dans les provinces et territoires présente certaines similitudes et est largement conforme aux directives du CCNI, avec quelques divergences. Des recherches supplémentaires sont nécessaires pour évaluer les programmes de vaccination contre la COVID-19 une fois qu'ils auront été mis en œuvre.
Cette oeuvre est mise à la disposition selon les termes de la licence internationale Creative Commons Attribution 4.0

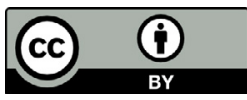

Affiliations

1 Faculty of Nursing, Université de l'Alberta, Edmonton, AB

2 School of Public Health, Université de l'Alberta,

Edmonton, $A B$

${ }^{3}$ Santé publique Ontario, Toronto, ON

${ }^{4}$ School of Public Health and Health Systems, Université de Waterloo, Waterloo, ON

${ }^{5}$ Département de pédiatrie, Université de Sherbrooke, Centre de Recherche du CHUS, Sherbrooke, QC

6 Université de la ColombieBritannique, Vancouver, BC

\section{*Correspondance :}

smacdon@ualberta.ca
Citation proposée : MacDonald SE, Sell H, Wilson S, Meyer SB, Gagneur A, Assi A, Sadarangani M et les membres du groupe d'étude COVImm. Analyse environnementale de la planification provinciale et territoriale aux fins des programmes de vaccination contre la COVID-19 au Canada. Relevé des maladies transmissibles au Canada 2021;47(5/6):312-9. https://doi.org/10.14745/ccdr.v47i56a07f

Mots-clés : COVID-19, vaccination, vaccin, pandémie, programme de vaccination 


\section{Introduction}

La course au développement de vaccins contre la maladie à coronavirus 2019 (COVID-19) est bien engagée, les premiers vaccins étant désormais autorisés au Canada (1). Les responsables canadiens de la santé publique sont confrontés au prochain défi majeur de la pandémie : la planification et la mise en œuvre des programmes de vaccination contre la COVID-19. La planification a été particulièrement difficile par rapport à d'autres vaccins, étant donné la rapidité avec laquelle le développement des vaccins a eu lieu, la nécessité de gérer plusieurs vaccins nouveaux, mais différents, et le besoin de vacciner une grande partie de la population pour entraver de manière significative la propagation du virus au sein de la population générale $(2,3)$. Comme l'approvisionnement initial en vaccins est limité $(3,4)$, une considération importante est la hiérarchisation des groupes cibles pour la vaccination contre la COVID-19. Le Comité consultatif national de l'immunisation (CCNI) a publié des directives définissant les populations clés pour l'administration initiale des vaccins $(3,5)$. Toutefois, c'est aux gouvernements provinciaux et territoriaux qu'il appartient, en dernier ressort, de suivre ou non ces directives et de déterminer la logistique des programmes de vaccination contre la COVID-19, y compris l'attribution des doses de vaccin, la livraison, l'entreposage, l'administration, le suivi et les rapports (6). À l'inverse, le rôle du gouvernement fédéral est d'approuver et d'acheter les vaccins, et de fournir des conseils sur leur utilisation (6).

Il est important de comprendre et de documenter les processus et les stratégies qui ont été employés par chacune des provinces et territoires dans leur planification de la vaccination contre la COVID-19. En reconnaissant l'éventail de stratégies, en mettant en évidence des approches nouvelles et innovantes et en tirant un apprentissage des approches réussies et non réussies, nous renforcerons notre capacité à répondre à des défis similaires auxquels nous serons sans doute confrontés à l'avenir. L'objectif de cette étude était donc d'utiliser des entretiens avec des informateurs clés afin de recueillir et synthétiser des informations concernant les programmes de vaccination contre la COVID-19 prévus dans chacune des provinces et territoires, y compris les considérations logistiques, les groupes prioritaires et le suivi de l'innocuité et de l'efficacité du vaccin.

\section{Méthodes}

Cette analyse environnementale pancanadienne implique des entrevues structurées avec des informateurs clés parmi les dirigeants de la santé publique des provinces et territoires du Canada. L'équipe de recherche était composée de chercheurs de six provinces et territoires, ainsi que d'utilisateurs de connaissances du secrétariat du $\mathrm{CCNI}$ et de trois services de santé provinciaux et territoriaux. Les utilisateurs de connaissances ont aidé dans le cadre du recrutement, ils ont cerné les sujets intéressants à inclure dans le guide d'entretien et ont reçu les résultats de l'étude pour qu'ils puissent s'y référer lors de la prise de décision. L'objectif de cette analyse était de recueillir et de synthétiser les points de vue des responsables de la santé publique qui participent activement à la planification des programmes d'immunisation provinciaux et territoriaux. Nous avons recruté des membres provinciaux et territoriaux du Comité canadien sur l'immunisation ( $\mathrm{CCl}$ ), et lorsqu'ils n'étaient pas disponibles pour participer, nous leur avons demandé de désigner un remplaçant. D'autres participants ont été identifiés par les membres de notre équipe de recherche, les services de santé provinciaux et territoriaux et le secrétariat du CCNI qui connaissent bien la direction des programmes de vaccination provinciaux et territoriaux. Nous avons communiqué avec les informateurs clés au moyen d'un premier courriel envoyé par le secrétariat du CCNI, les invitant à participer à l'étude. Les personnes intéressées ont reçu par courriel une fiche d'information et un formulaire de consentement. Pour optimiser le taux de réponse, nous avons envoyé jusqu'à deux rappels par courriel. Certains participants ont été identifiés par un échantillonnage en boule de neige, les participants à l'étude ayant suggéré d'autres informateurs clés. Les entretiens ont eu lieu entre août et octobre 2020, avant la publication des orientations préliminaires du CCNI (3) et l'approbation des vaccins contre la COVID-19 au Canada. Les entretiens (d'une durée de 35 à 60 minutes) ont été menés par les membres de l'équipe de recherche (H. S., A. A., M. K.) par téléconférence.

Les questions de l'entretien portaient sur les principaux sujets liés à la vaccination contre la COVID-19, établis dans la littérature scientifique et les articles de presse, et complétés par les contributions des experts en vaccination de l'équipe de recherche et des utilisateurs de connaissances, y compris le secrétariat du CCNI (voir matériel supplémentaire). Le guide d'entretien structuré comprenait principalement des questions ouvertes sur les sujets suivants : les facteurs uniques à prendre en compte dans la planification du programme de vaccination contre la COVID-19, le degré de confiance accordé aux recommandations du CCNI, I'utilisation d'un cadre de hiérarchisation géographique pour l'allocation des vaccins, les groupes cibles à privilégier pour la première phase de la vaccination et les plans de surveillance de l'innocuité et de l'efficacité des vaccins. Le guide d'entretien a été revu et corrigé par des experts en immunisation et a fait l'objet d'un essai pilote auprès d'une personne travaillant à la planification du programme provincial d'immunisation, mais n'ayant pas participé à l'étude, afin de vérifier la validité apparente et le contenu, la fluidité et la compréhension. L'inclusion de multiples perspectives au sein des provinces et territoires, et entre eux, a renforcé la crédibilité des résultats. Le guide a été partagé avec les participants avant l'entretien. L'approbation éthique de cette étude a été obtenue auprès du Comité de l'éthique de la recherche en santé de I'Université de l'Alberta. 
Les entretiens ont été enregistrés et transcrits mot à mot par un membre de l'équipe de recherche et toute information permettant d'identifier les personnes a été supprimée. Le même membre de l'équipe a ensuite codé et catégorisé les réponses des participants. Compte tenu de la nature très structurée des entretiens, l'analyse a nécessité peu d'interprétation subjective. Cependant, pour garantir la rigueur, le codage et la catégorisation ont été validés par trois autres membres de l'équipe afin de veiller à ce qu'ils reflètent fidèlement les réponses des participants et en soient pleinement représentatifs. Une analyse descriptive du nombre de réponses a été réalisée à I'aide de Microsoft Excel. Les réponses des participants ont été synthétisées et présentées par provinces et territoires.

\section{Résultats}

Des courriels d'invitation du CCNI ont été envoyés à 35 participants potentiels : 13 ont accepté de participer, un a refusé et 21 n'ont pas répondu. Douze participants ont été recrutés parmi d'autres participants, cinq par le biais de références et sept se sont joints aux entretiens de leurs collègues. Par conséquent, certains entretiens comportaient plus d'un participant. Au total, il y a eu 18 entretiens avec 25 participants provenant de 11 des 13 provinces et territoires. Le tableau 1 présente les données démographiques de l'échantillon de l'étude.

Tableau $1:$ Informations démographiques de l'échantillon de l'étude $(\mathrm{N}=25)^{\mathrm{a}}$

\begin{tabular}{|c|c|}
\hline Caractéristique & Nombre de participants, n \\
\hline \multicolumn{2}{|l|}{ Province/territoire } \\
\hline Colombie-Britannique & 1 \\
\hline Alberta & 4 \\
\hline Saskatchewan & 3 \\
\hline Manitoba & 4 \\
\hline Ontario & 3 \\
\hline Québec & 3 \\
\hline Terre-Neuve-et-Labrador & 1 \\
\hline Nouvelle-Écosse & 3 \\
\hline Nouveau-Brunswick & 0 \\
\hline Île-du-Prince-Édouard & 1 \\
\hline Nunavut & 1 \\
\hline Territoires du Nord-Ouest & 1 \\
\hline Yukon & 0 \\
\hline \multicolumn{2}{|l|}{ Perspective } \\
\hline Provinciale/territoriale & 12 \\
\hline Régionale/municipale & 9 \\
\hline Les deux & 4 \\
\hline \multicolumn{2}{|l|}{ Titre du poste } \\
\hline $\begin{array}{l}\text { Directeur de l'immunisation ou du } \\
\text { contrôle des maladies transmissibles }\end{array}$ & 2 \\
\hline
\end{tabular}

Tableau 1 : Informations démographiques de l'échantillon de l'étude $(\mathrm{N}=25)^{\text {a }}$ (suite)

\begin{tabular}{|c|c|}
\hline Caractéristique & Nombre de participants, n \\
\hline \multicolumn{2}{|l|}{ Titre du poste (suite) } \\
\hline $\begin{array}{l}\text { Responsable du programme ou de } \\
\text { la politique d'immunisation }\end{array}$ & 7 \\
\hline Médecin-conseil en santé publique & 5 \\
\hline $\begin{array}{l}\text { Consultant en santé publique ou en } \\
\text { médecine }\end{array}$ & 3 \\
\hline Analyste politique & 2 \\
\hline $\begin{array}{l}\text { Spécialiste de la santé publique ou } \\
\text { des maladies transmissibles }\end{array}$ & 2 \\
\hline Autre & 4 \\
\hline
\end{tabular}

\section{Facteurs uniques pour les programmes de vaccination contre la COVID-19}

Un large éventail de facteurs propres à la planification des programmes de vaccination contre la COVID-19 a été établi (voir tableau 2). Les participants d'un peu plus de la moitié des provinces et territoires $(n=7)$ ont indiqué la nécessité de hiérarchiser les groupes cibles pour les premières phases de la vaccination. De nombreuses provinces et territoires $(n=5)$ ont également souligné la possibilité d'avoir des critères d'admissibilité différents pour chaque vaccin (i.e. si un vaccin est plus efficace chez les adultes plus âgés), ce qui pourrait avoir une incidence sur l'ordre des groupes prioritaires.

\section{Tableau 2 : Facteurs uniques des provinces et territoires qu'il est prévu de prendre en compte pour les programmes de vaccination contre la COVID-19 $(\mathrm{N}=11)$}

\begin{tabular}{|c|c|}
\hline Facteur unique & Nombre de $\mathrm{PT}^{\mathrm{a}}$, $\mathbf{n}$ \\
\hline \multicolumn{2}{|l|}{ Groupes prioritaires } \\
\hline Hiérarchisation des groupes cibles & 7 \\
\hline Critères d'éligibilité divergents & 5 \\
\hline Équité dans la prestation & 1 \\
\hline \multicolumn{2}{|l|}{ Engagement du public } \\
\hline $\begin{array}{l}\text { Perception du vaccin par le public, y compris } \\
\text { I'hésitation à se faire vacciner }\end{array}$ & 6 \\
\hline Communication claire avec le public & 3 \\
\hline \multicolumn{2}{|l|}{ Logistique et approvisionnement } \\
\hline $\begin{array}{l}\text { Logistique, entreposage, gestion de la chaîne } \\
\text { du froid }\end{array}$ & 4 \\
\hline $\begin{array}{l}\text { Approvisionnement limité en vaccins, } \\
\text { disponibilité des vaccins }\end{array}$ & 3 \\
\hline $\begin{array}{l}\text { Disponibilité de l'EPI et des autres fournitures } \\
\text { de vaccination (autres que le vaccin lui-même) }\end{array}$ & 3 \\
\hline Distribution de vaccins & 2 \\
\hline Questions relatives aux ressources (en général) & 2 \\
\hline Achat de vaccins & 1 \\
\hline
\end{tabular}


Tableau 2 : Facteurs uniques des provinces et territoires qu'il est prévu de prendre en compte pour les programmes de vaccination contre la COVID-19 ( $N=11$ ) (suite)

\begin{tabular}{|c|c|}
\hline Facteur unique & Nombre de $\mathrm{PT}^{\mathrm{a}}$, $\mathrm{n}$ \\
\hline \multicolumn{2}{|l|}{ Livraison } \\
\hline $\begin{array}{l}\text { Restrictions liées à la COVID-19, mesures de } \\
\text { santé publique, EPI }\end{array}$ & 4 \\
\hline $\begin{array}{l}\text { Fournisseur de vaccins (e.g. médecins, } \\
\text { pharmaciens, santé publique) }\end{array}$ & 4 \\
\hline $\begin{array}{l}\text { Prestation sur rendez-vous par rapport aux } \\
\text { cliniques de masse }\end{array}$ & 3 \\
\hline $\begin{array}{l}\text { Nécessité de vacciner tout le monde, grand } \\
\text { nombre de personnes }\end{array}$ & 3 \\
\hline $\begin{array}{l}\text { Moins de ressources humaines en raison du } \\
\text { redéploiement dû à la COVID-19 }\end{array}$ & 3 \\
\hline Formation destinée aux prestataires & 2 \\
\hline $\begin{array}{l}\text { Incertitude, manque d'informations aux fins de } \\
\text { la planification }\end{array}$ & 2 \\
\hline \multicolumn{2}{|l|}{ Caractéristiques des vaccins } \\
\hline Possibilité d'avoir besoin de plus d'une dose & 4 \\
\hline Innocuité des vaccins & 3 \\
\hline Faire face à un nouveau vaccin & 3 \\
\hline $\begin{array}{l}\text { Considérations relatives à la voie } \\
\text { d'administration }^{\text {'adm }}\end{array}$ & 3 \\
\hline Possibilité d'avoir plus d'un vaccin & 2 \\
\hline $\begin{array}{l}\text { Rapidité avec laquelle le développement des } \\
\text { vaccins se fait }\end{array}$ & 2 \\
\hline
\end{tabular}

Certains participants des provinces et territoires ont également discuté des facteurs liés à l'engagement du public, notamment le fait d'avoir une communication claire avec le public concernant les implications en matière d'innocuité, les critères d'admissibilité et les groupes prioritaires ( $n=3$ provinces et territoires). De même, six provinces et territoires ont souligné la nécessité de gérer la perception des vaccins contre la COVID-19 par le public. Plus précisément, une province ou un territoire avait l'impression que l'hésitation relative aux vaccins contre la COVID-19 serait plus grande que pour les vaccins précédents.

Les provinces et territoires ont également discuté des facteurs uniques liés à la logistique et à l'approvisionnement des vaccins contre la COVID-19. Quatre provinces et territoires ont souligné les exigences uniques d'entreposage de certains vaccins, et certaines provinces et territoires ont déclaré qu'il était peu probable que tous les fournisseurs aient actuellement la capacité d'entreposer les vaccins à la température appropriée. D'autres ont fait remarquer que l'approvisionnement en vaccins $(n=3)$ et en autres fournitures de vaccination $(n=3)$ sera probablement limité.
La planification de l'administration des vaccins contre la COVID-19 s'annonçait difficile, certaines provinces et territoires $(n=4)$ ayant déclaré ne pas savoir quels prestataires administreraient les vaccins (e.g. santé publique, médecins, pharmaciens), ni s'il s'agirait de cliniques sur rendez-vous ou de cliniques de masse $(n=3)$. De même, quatre provinces et territoires ont mentionné la nécessité d'adapter les cliniques de vaccination aux recommandations relatives à la COVID-19, notamment en ce qui concerne la distance physique, l'équipement de protection individuelle, l'aménagement, la circulation à sens unique et la ventilation. Une province ou territoire a mentionné qu'elle avait inclus des ingénieurs industriels dans son équipe de planification pour tenir compte de ces facteurs. Une liste complète des réponses des provinces et territoires est fournie dans le tableau 2.

\section{Recours aux recommandations du CCNI}

Presque toutes les provinces et territoires $(n=10)$ ont indiqué qu'ils s'appuieraient probablement sur les recommandations du CCNI concernant les groupes cibles pour planifier leurs stratégies de vaccination contre la COVID-19. Une province ou un territoire a indiqué qu'il serait plus probable qu'il se fie aux recommandations de son comité d'immunisation provincial/ territorial.

\section{Classement des groupes prioritaires}

Les participants ont été invités à classer leurs cinq groupes prioritaires, le rang 1 représentant le groupe qui devrait être vacciné en premier contre la COVID-19. Aux fins du rapport, nous avons utilisé le classement du répondant de chaque province et territoire qui avait le plus de perspective interprovinciale en fonction de son poste et s'il a déclaré avoir une perspective provinciale plutôt que régionale/municipale. Une province ou un territoire n'a pas répondu, ce qui donne un total de 10 provinces et territoires. Toutes les provinces et territoires ont classé les résidents des établissements de soins de longue durée $(n=10)$ et les travailleurs de la santé $(n=10)$ parmi les cinq groupes prioritaires pour la vaccination contre la COVID-19. Plus précisément, six provinces et territoires ont indiqué que les résidents des établissements de soins de longue durée constituaient une priorité absolue, trois ont indiqué les travailleurs de la santé et un a indiqué les personnes âgées. Trois provinces et territoires ont classé les travailleurs de la santé au deuxième rang, suivis des résidents de centres de soins de longue durée $(n=2)$, des personnes atteintes de maladies chroniques $(n=2)$, des personnes âgées $(n=2)$ et des travailleurs essentiels $(n=1)$. Les groupes classés au troisième rang comprenaient les personnes âgées $(n=3)$, les résidents de centres de soins de longue durée $(n=2)$, les travailleurs de la santé $(n=1)$, les personnes atteintes de maladies chroniques $(n=1)$, les personnes d'ascendance autochtone $(n=1)$, les personnes défavorisées sur le plan socio-économique $(n=1)$ et les personnes vivant dans des communautés éloignées $(n=1)$. La figure 1 présente un résumé complet des classements provinces et territoires. 
Figure 1 : Choix des groupes prioritaires des provinces et territoires à inclure dans leurs cinq premiers groupes à envisager pour les premières phases de la vaccination en cas d'approvisionnement limité en vaccins $(\mathbf{N}=10)^{\mathrm{a}, \mathrm{b}}$



a Une province ou un territoire a choisi de ne pas répondre à cette question

b Pour ceux qui ont choisi les personnes âgées $(n=8)$, sept ont indiqué qu'ils cibleraient les personnes âgées de 65 ans et plus, tandis qu'un a indiqué qu'il ciblerait les personnes âgées de 60 ans et plus

\section{Utilisation d'un cadre de hiérarchisation géographique}

Aucune des provinces et territoires n'avait de plan ferme pour un cadre de hiérarchisation géographique fondé sur l'incidence de la maladie (i.e. que les groupes cibles dans les zones à forte incidence de COVID-19 sont prioritaires par rapport aux groupes cibles dans les zones à faible incidence). La majorité des provinces et territoires $(n=7)$ étaient ouverts à cette approche si le CCNI le conseillait ( $n=1)$, ou si les caractéristiques du vaccin $(n=1)$ ou le nombre de doses disponibles $(n=3)$ le justifiaient.

Trois provinces et territoires s'opposaient à l'utilisation d'un cadre de hiérarchisation géographique en raison de préoccupations quant à l'équité de cette approche $(n=1)$ ou en raison de la petite taille de leur territoire ou de la densité de leur population $(n=2)$. Une province ou un territoire ne savait pas s'il prévoyait utiliser un cadre de hiérarchisation géographique.

\section{Contrôle de l'innocuité et de l'efficacité des vaccins}

En ce qui concerne la surveillance de l'innocuité des vaccins après leur mise en marché, la plupart des provinces et territoires $(n=9)$ prévoyaient d'effectuer leur surveillance habituelle des effets indésirables, tandis que certains $(n=3)$ en prévoyaient une surveillance accrue (voir le tableau 3 ). Certaines provinces et territoires $(n=4)$ prévoient que cela sera fait par des comités et des groupes fédéraux/provinciaux/territoriaux. En ce qui concerne la surveillance de l'efficacité des vaccins après leur mise en marché, certaines provinces et territoires $(n=3)$ prévoient que leurs services de santé publique provinciaux et territoriaux s'en chargeront, et un nombre similaire $(n=3)$ affirme que cette information sera recueillie de façon routinière. $D^{\prime}$ autres $(n=3)$
Tableau 3 : Approche prévue par les provinces et territoires pour le suivi de l'innocuité et de l'efficacité du vaccin contre la COVID-19 ( $N=11)$

\begin{tabular}{|c|c|}
\hline Approche planifiée & Nombre de PTa, $n$ \\
\hline \multicolumn{2}{|l|}{ Innocuité } \\
\hline Rapports réguliers sur les effets indésirables & 9 \\
\hline Surveillance renforcée des effets indésirables & 3 \\
\hline $\begin{array}{l}\text { Recours à des comités et groupes fédéraux/ } \\
\text { provinciaux/territoriaux (e.g. CIC, RCRICV) }\end{array}$ & 4 \\
\hline Recours aux recommandations du CCNI & 1 \\
\hline Indécis & 1 \\
\hline Ne sait pas & 2 \\
\hline
\end{tabular}

\section{Efficacité}

Recours à la santé publique provinciale (e.g.

équipes de surveillance)

Recours à des chercheurs et des organismes de recherche

Recours au CCNI ou à d'autres orientations nationales

Collecte des informations de suivi de routine (e.g. le nombre de clients dont le test est positif après la vaccination, la couverture vaccinale)

\begin{tabular}{|l|r|}
\hline Indécis & 2 \\
\hline Ne sait pas & 1 \\
\hline Pas de réponse & 2 \\
\hline
\end{tabular}

Abréviations : $\mathrm{ClC}$, Comité canadien sur l'immunisation; $\mathrm{CCNI}$, Comité consultatif national de I'immunisation; PT, province/territoire; RCRICV, Réseau canadien des registres d'immunisation et de couverture vaccinale

a Certaines réponses des provinces et territoires entrent dans plus d'une des catégories ci-dessus

s'attendent à ce que cela soit fait par des chercheurs ou des organismes de recherche.

\section{Discussion}

Bien que les classements provinciaux et territoriaux des groupes prioritaires potentiels aient été recueillis avant la publication des documents d'orientation du CCNI, les classements globaux des provinces et territoires s'alignent quelque peu sur les recommandations du CCNI. Plus précisément, les groupes les mieux classés dans cette étude sont les travailleurs de la santé et les résidents de centres de soins de longue durée, suivis des personnes souffrant de maladies chroniques et des personnes âgées. Les recommandations les plus récentes du CCNI donnent la priorité aux travailleurs de la santé, aux résidents et au personnel des établissements de soins de longue durée, aux personnes âgées de 70 ans et plus (celles de 80 ans et plus étant prioritaires) et aux adultes des communautés autochtones (5). La différence remarquable entre les classements des provinces et territoires dans notre étude et les recommandations du $\mathrm{CCNI}$ est que moins de la moitié des provinces et territoires 
ont classé les communautés autochtones parmi les cinq premiers groupes prioritaires, et que les personnes souffrant d'affections chroniques (classées au troisième rang par la plupart des provinces et territoires) n'ont pas été incluses dans les orientations les plus récentes du CCNI sur les premières phases de la vaccination (5).

Une considération commune aux provinces et territoires était la perception négative potentielle des vaccins contre la COVID-19 par le public. De nombreuses provinces et territoires ont reconnu le rôle important que la santé publique aura à jouer dans l'élaboration de stratégies de communication pour contrer ces préoccupations. Selon une enquête de Statistique Canada réalisée en juin 2020, 76,5 \% des Canadiens seraient très susceptibles ou assez susceptibles de se faire vacciner contre la COVID-19 lorsqu'il sera disponible (7), mais les données d'une enquête nationale Leger réalisée en novembre 2020 estiment que ce chiffre est de $65 \%$ (8).

Les provinces et territoires ont également souligné les difficultés logistiques liées à l'administration du vaccin et la nécessité de veiller à ce que les cliniques de vaccination suivent les recommandations de santé publique en matière de distanciation, d'utilisation d'équipement de protection individuelle, de désinfection et de ventilation, etc. De nombreuses provinces et territoires considéraient le programme de lutte contre la grippe saisonnière 2020-2021 comme un essai sur la façon dont l'administration du vaccin contre la COVID-19 pourrait se dérouler. À la suite de la pandémie de grippe H1N1, il a été remarqué que des programmes de vaccination contre la grippe qui fonctionnent bien sont essentiels pour garantir la disponibilité d'une infrastructure adéquate pour la réponse à une vaccination pandémique (9). Au début de la pandémie, le CCNI a fourni des conseils sur les stratégies de distribution du vaccin antigrippal pendant la pandémie (10).

Le fait d'avoir une approche unifiée de la vaccination contre la COVID-19 au Canada peut être bénéfique pour fournir un message public cohérent et clarifier pourquoi certains groupes prioritaires ont été sélectionnés pour les premières phases de la vaccination. Les stratégies de communication publique sont importantes pour prévenir l'hésitation et la méfiance à l'égard des vaccins (9). En outre, une approche unifiée de la vaccination peut améliorer l'équité et permettre de réaliser des économies (11). Les critiques de la variabilité provinciale et territoriale de longue date concernant les programmes et les calendriers de vaccination au Canada ont fait valoir que le manque d'uniformité dans l'admissibilité et les modes de prestation entraîne des incohérences dans les messages publics, ce qui peut miner la confiance du public lorsque la justification des différences n'est pas claire $(11,12)$. Inversement, la diversité entre les provinces et territoires permet de s'adapter aux circonstances uniques de chaque juridiction, étant donné la variation de la géographie, de la population et des cas de COVID-19 entre les provinces et territoires (13). Même si les provinces et territoires vont inévitablement élaborer leurs propres plans de vaccination contre la COVID-19, les résultats de cette étude suggèrent qu'il y aura probablement de nombreuses similitudes.

\section{Forces et faiblesses}

La force de cette étude réside dans la grande variété de perspectives obtenues sur la planification du programme de vaccination contre la COVID-19 dans la plupart des provinces et territoires. De même, le recours à des entretiens avec des informateurs clés nous a permis de recueillir des points de vue approfondis sur la planification du programme de vaccination contre la COVID-19 dans chaque province et territoire. Cependant, étant donné que seules quelques personnes choisies ont été interrogées dans chaque province et territoire, les perspectives recueillies ne sont pas représentatives de l'ensemble des provinces et territoires. En outre, il peut y avoir des variations dans les perspectives individuelles au sein d'un même PT, bien que les perspectives partagées soient très cohérentes au sein d'une province ou d'un territoire donné. La généralisation peut être limitée en raison de la petite taille de l'échantillon et de l'échantillonnage non aléatoire. Les entretiens ont été réalisés à une période où la planification de la vaccination contre la COVID-19 en était à ses débuts. II sera intéressant de voir si les premiers plans ont changé depuis la publication des documents d'orientation du CCNI $(3,5)$.

\section{Implications}

La mise en œuvre de programmes de vaccination contre la COVID-19 au Canada en est à ses débuts. Il est possible d'approfondir les résultats de cette étude par le biais d'une variété d'avenues de recherche, y compris l'évaluation du plan de vaccination contre la COVID-19 finalisé de chaque province et territoire, et la façon dont les variations dans les programmes de vaccination affectent finalement l'adoption du vaccin et son efficacité dans chaque provinces et territoires.

Cette étude s'ajoute à la littérature existante en synthétisant les perspectives de santé publique provinciaux et territoriaux sur les programmes de vaccination contre la COVID-19 à un stade de planification. Les résultats peuvent informer les décideurs politiques et les planificateurs de programmes et peuvent aider le CCNI dans le développement futur de directives nationales. Nous prévoyons que l'information contenue dans cette étude permettra aux provinces et territoire d'apprendre les uns des autres en comparant leur approche de la vaccination contre la COVID-19 avec d'autres à travers le Canada.

\section{Conclusion}

Les résultats des entrevues avec les informateurs clés montrent que les provinces et territoires canadiens font face à des défis similaires dans la planification de la vaccination contre la COVID-19. La majorité d'entre eux s'appuieront sur les recommandations du $\mathrm{CCNI}$ concernant la manière de répartir l'approvisionnement limité en vaccins. D'autres recherches sont nécessaires pour évaluer les programmes provinciaux et 
territoriaux de vaccination contre la COVID-19 une fois qu'ils seront mis en œuvre.

\section{Déclaration des auteurs}

S. MacDonald - Conceptualisation, méthodologie, acquisition de fonds, supervision, analyse formelle, rédaction (version originale, et révision et édition)

H. S. - Conceptualisation, enquête, collecte de données, analyse formelle, rédaction (version originale, révision et édition) S. W. - Conceptualisation, méthodologie, rédaction (révision et édition)

S. Meyer - Conceptualisation, méthodologie, rédaction (révision et édition)

A. G. - Conceptualisation, méthodologie, acquisition de fonds, rédaction (révision et édition)

A. A. - Conceptualisation, enquête, conservation des données, supervision, administration du projet, analyse formelle, rédaction (révision et édition)

M. S. - Conceptualisation, méthodologie, rédaction (révision et édition)

Le contenu de l'article et les points de vue qui y sont exprimés n'engagent que les auteurs et ne correspondent pas nécessairement à ceux du gouvernement du Canada.

\section{Intérêts concurrents}

S. MacDonald est soutenu par une bourse salariale du Programme canadien de cliniciens-chercheurs en santé infantile. M. S. est soutenu par des bourses salariales de la BC Children's Hospital Foundation, du Canadian Child Health Clinician Scientist Program et de la Michael Smith Foundation for Health Research. M. S. a été chercheur sur des projets financés par GlaxoSmithKline, Merck, Pfizer, Sanofi-Pasteur, Seqirus, Symvivo et VBI Vaccines. Tous les fonds ont été versés à son institut, et il n'a reçu aucun paiement personnel.

\section{Remerciements}

Cette étude faisait partie d'un projet plus vaste mené par le groupe d'étude COVImm, qui comprenait les auteurs nommés, ainsi que : M. Tunis, K. Benzies, J. Bettinger, M. Driedger, E. Dubé, R. Humble, M. Kiely, N. MacDonald, E. Rafferty et J. Robinson. Nous remercions tout particulièrement M. Kiely pour son aide lors des entretiens.

\section{Financement}

Ce travail a été financé par les Instituts de recherche en santé du Canada.

\section{Matériel supplémentaire}

Study interview guide (en anglais seulement)

\section{Références}

1. Gouvernement du Canada. Vaccins contre la COVID-19 : Vaccins autorisés. Ottawa (ON): Gouvernement du Canada; (modifié 2021; accédé 2021-03-05). https://www.canada.ca/fr/ sante-canada/services/medicaments-produits-sante/covid19industrie/medicaments-vaccins-traitements/vaccins.html

2. MacDonald NE, Comeau JL, Dubé È, Bucci LM. COVID-19 and missed routine immunizations: designing for effective catch-up in Canada. Can J Public Health 2020;111(4):469-72. DOI PubMed

3. Ismail SJ, Zhao L, Tunis MC, Deeks SL, Quach C; National Advisory Committee on Immunization. Key populations for early COVID-19 immunization: preliminary guidance for policy. CMAJ 2020;192(48):E1620-32. DOI PubMed

4. Organisation de la mondiale de la Santé. Feuille de route du SAGE de I'OMS pour l'établissement des priorités concernant I'utilisation des vaccins anti-Covid-19 dans un contexte d'approvisionnement limité. Genève, Suisse : OMS; 2020 (accédé 2021-03-05). https://www.who.int/fr/publications/m/ item/who-sage-roadmap-for-prioritizing-uses-of-covid-19vaccines-in-the-context-of-limited-supply

5. Comité consultatif national de l'immunisation (CCNI). Orientations sur l'administration prioritaire des premières doses du vaccin contre la COVID-19. Ottawa (ON) : Gouvernement du Canada; 2020 (accédé 2020-12-14). https://www.canada. $\mathrm{ca} / \mathrm{fr} /$ sante-publique/services/immunisation/comite-consultatifnational-immunisation-ccni/orientations-administrationprioritaire-premieres-doses-vaccine-covid-19.html

6. Gouvernement du Canada. Immunisation contre la COVID-19 : Énoncé de principes communs fédéral, provincial et territorial. Ottawa (ON) : Gouvernement du Canada; 2020 (accédé 2021-03-05). https://www.canada.ca/fr/sante-publique/ services/maladies/2019-nouveau-coronavirus/reponse-canada/ immunisation-covid-19-enonce-principes-communs-federalprovincial-territorial.html

7. Frank K, Arim R. La volonté des Canadiens de se faire vacciner contre la COVID-19 : différences entre les groupes et raisons de la réticence à la vaccination. Ottawa $(\mathrm{ON})$ : Statistique Canada; 2020 (accédé 2020-12-11). https://www150.statcan.gc.ca/n1/ pub/45-28-0001/2020001/article/00073-fra.htm

8. Leger. North American tracker. Montreal (QC): Leger; 2020 (accédé 2020-12-11). https://leger360.com/wp-content/ uploads/2020/11/Legers-North-American-Tracker-November30th-2020-min.pdf?x43558

9. Sultana J, Mazzaglia G, Luxi N, Cancellieri A, Capuano A, Ferrajolo C, de Waure C, Ferlazzo G, Trifirò G. Potential effects of vaccinations on the prevention of COVID-19: rationale, clinical evidence, risks, and public health considerations. Expert Rev Vaccines 2020;19(10):919-36. DOI PubMed

10. Comité consultatif national de l'immunisation (Une déclaration d'un comité consultatif). Chapitre sur la grippe du Guide canadien d'immunisation et Déclaration sur la vaccination antigrippale pour la saison 2020-2021. Ottawa (ON) : ASPC; 2020 (accédé 2020-12-15). https://www.canada.ca/content/ dam/phac-aspc/documents/services/publications/healthy-living/ canadian-immunization-guide-statement-seasonal-influenzavaccine-2020-2021/naci-2020-2021-seasonal-influenza-stmt-fra. pdf 
11. Macdonald N, Bortolussi R. A harmonized immunization schedule for Canada: A call to action. Paediatr Child Health 2011;16(1):29-31. DOI PubMed

12. A patchwork policy: vaccination in Canada. CMAJ 2003;168(5):533. PubMed
13. Gouvernement du Canada. Mise à jour quotidienne sur I'épidémiologie de la COVID-19. Ottawa (ON) : Gouvernement du Canada; 2021 (accédé 2021-03-09). https://sante-infobase. canada.ca/covid-19/resume-epidemiologique-cas-covid-19.html

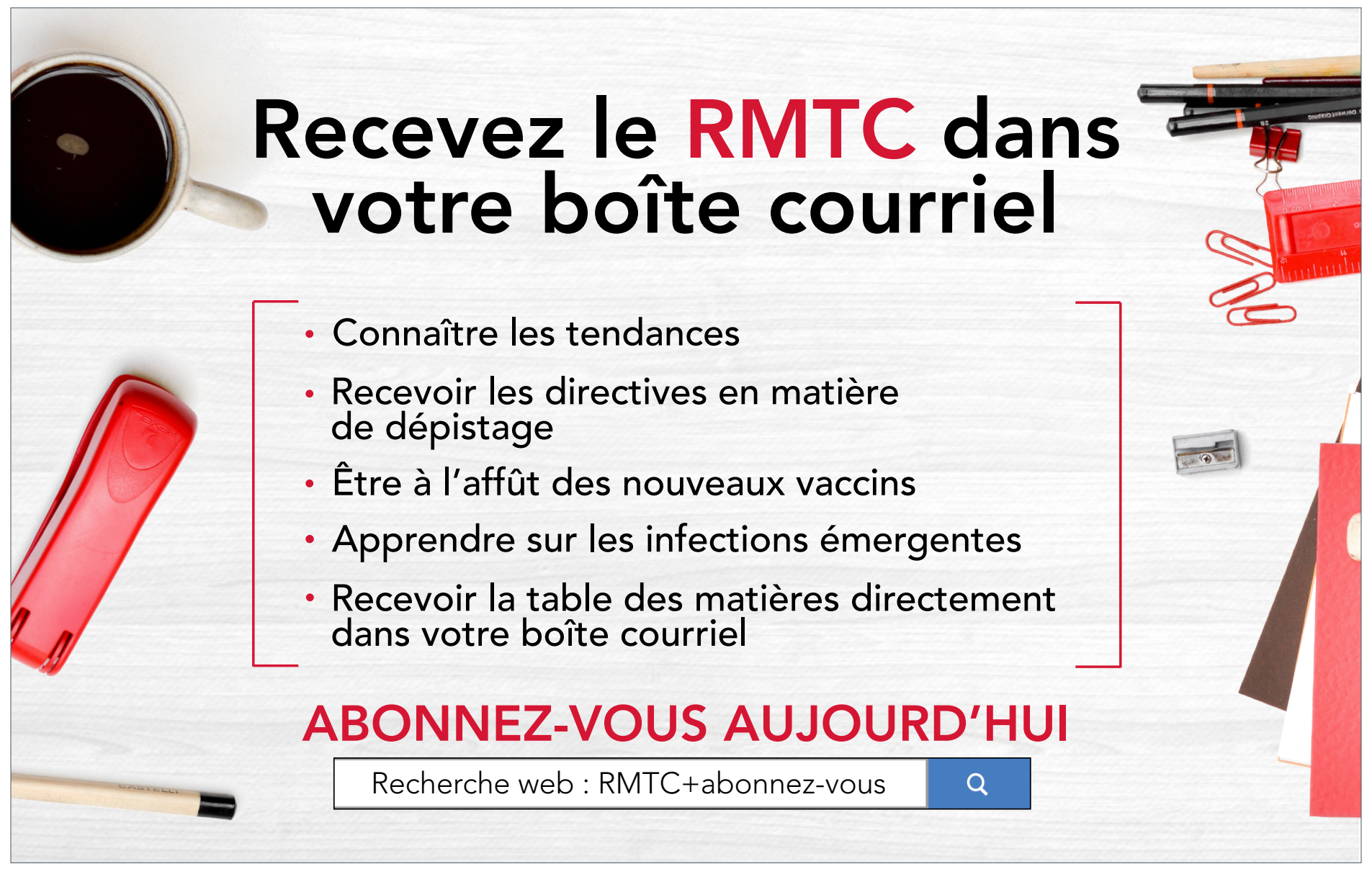

